\title{
ON COMPOSITIONS OF ISOMETRIC IMMERSIONS
}

\author{
MARCOS DAJCZER \& RUY TOJEIRO
}

The starting goal of this work was to understand local and global isometric immersions with low codimension of the $n$-dimensional round sphere $S^{n}$ into Euclidean space $\mathbf{R}^{n+p}$. For codimension $p=2$ and dimension $n \geq 4$, this problem was considered by O'Neill [16], Erbacher [11], Henke [12], Whitt [19], and Moore [15]. Erbacher proved that in a neighborhood of a nonumbilical point the immersion is necessarily a composition of the standard inclusion of $S^{n}$ into $\mathbf{R}^{n+1}$ with a local isometric immersion of $\mathbf{R}^{n+1}$ into $\mathbf{R}^{n+2}$. A simple example provided by Henke shows that for an umbilical point this may no longer be the case.

In fact, earlier work due to O'Neill strongly suggested that a result similar to Erbacher's should hold up to codimension $p=n-2$ under suitable regularity assumptions which extend the umbilical-free hypothesis. In the process of getting a solution to this problem we realized the possibility of setting a more general rigidity question. Namely, in $\S 1$, we provide conditions on isometric immersions $f: M^{n} \rightarrow Q_{c}^{n+1}$ and $g: M^{n} \rightarrow Q_{c}^{n+p}$, $p \geq 2$, which imply that $g$ is necessarily (locally or globally) a composition. Here and throughout the paper, $M^{n}$ is a connected $n$-dimensional Riemannian manifold, $Q_{c}^{N}$ denotes a complete simply connected Riemannian manifold of constant sectional curvature $c$, and $g$ is a composition when there exists an isometric immersion $h: U \rightarrow Q_{c}^{n+p}$ of an open subset $U \subset Q_{c}^{n+1}$ containing $f(M)$ so that $g=h \circ f$.

Let us denote by $\rho_{f}(x)$ the number of nonzero principal curvatures of the hypersurface $f$ at $x \in M$. Without making any regularity assumption we prove the following result.

Theorem 1. Let $f: M^{n} \rightarrow Q_{c}^{n+1}$ and $g: M^{n} \rightarrow Q_{c}^{n+p}, p \geq 2$, be isometric immersions with $\rho_{f}(x) \geq p+2$ everywhere. If $p \geq 6$, assume further that $M^{n}$ does not contain an open $(n-p+2)$-ruled subset for both $f$ and $g$. Then there exists an open and dense subset $V \subset M$ so that $\left.g\right|_{V}$ is locally a composition.

Received March 5, 1991. 
The restriction on the codimension in the above result cannot be weakened. In a forthcoming paper (see [6]) we show that there exists a large family of local isometric immersions of $S^{n}$ into $\mathbf{R}^{2 n-1}$ which are nowhere compositions.

In fact, by imposing further conditions in Theorem 1, we are able to prove a global and more general result. First we need some terminology. Recall that the first normal space $N_{1}^{g}(x)$ of an isometric immersion $g$ at a point $x \in M$ is the normal subspace defined as

$$
N_{1}^{g}(x)=\operatorname{span}\left\{\alpha_{g}(X, Y): X, Y \in T_{x} M\right\},
$$

where $\alpha_{g}$ stands for the vector-valued second fundamental form of the immersion. From now on set $s_{g}(x)=\operatorname{dim} N_{1}^{g}(x)$. We say that $g$ is 1-regular if $s_{g}(x)$ is constant on $M$.

Theorem 2. Let $f: M^{n} \rightarrow Q_{c}^{n+1}$ and $g: M^{n} \rightarrow Q_{c}^{n+p}, p \geq 2$, be isometric immersions where $g$ is 1-regular and $\rho_{f}(x) \geq s_{g}+2$ everywhere.

(a) Then $\alpha_{g}$ decomposes orthogonally and smoothly as $\alpha_{g}=\alpha_{f} \oplus \gamma$, where for all $x \in M$, the nullity space of $\gamma(x)$ verifies $\operatorname{dim} N(\gamma(x)) \geq$ $n-s_{g}+1$.

(b) Suppose now that $f$ is an embedding and $\operatorname{dim} N(\gamma(x))=k$ is constant. If $s_{g} \geq 6$ and $k \geq n-s_{g}+2$, assume further that $M^{n}$ does not contain $a k$-ruled open subset for both $f$ and $g$. Then $g$ is (globally) $a$ composition.

Our assumptions that $g$ is 1-regular and that $\operatorname{dim} N(\gamma(x))=k$ is constant are precisely the regularity conditions considered by O'Neill.

The remaining of the paper is devoted to several applications where a "hidden composition" turns out to be the main tool. In $\S 2$, we deal with the problem of whether a Riemannian manifold $M^{n}$ can be isometrically immersed into two space forms $Q_{c}^{n+1}$ and $Q_{\tilde{c}}^{n+p}$ with $c<\tilde{c}$. This situation was first considered by do Carmo and Dajczer ([7], see also [1]). Given $f: M^{n} \rightarrow Q_{c}^{n+1}$ and $g: M^{n} \rightarrow Q_{\tilde{c}}^{n+p}$ they showed that if $s_{g}(x) \leq n-3$ at $x \in M$, then there exists an umbilical subspace $U(x) \subset T_{x} M$ for both immersions with $\operatorname{dim} U(x) \geq n-s_{g}(x)$. It is a well-known fact (see [18]) that the umbilical subspaces form an integrable smooth distribution with umbilical leaves on any open subset of $M$ where they have constant dimension. In particular, a complete understanding of the geometric origin of the common umbilical foliation follows from our next result.

Theorem 3. Suppose that $M^{n}, n \geq 4$, admits isometric immersions $f: M^{n} \rightarrow Q_{c}^{n+1}$ and $g: M^{n} \rightarrow Q_{\tilde{c}}^{n+p}, c<\tilde{c}$, with $s_{g}(x) \leq n-3$ everywhere. Set $G=i \circ g$, where $i$ is the umbilical inclusion of $Q_{\tilde{c}}^{n+p}$ into 
$Q_{c}^{n+p+1}$. Then there exists an open dense subset $V \subset M$ so that $\left.G\right|_{V}$ is locally a composition.

By the above, the immersions $f$ and $g$ can always be locally produced as the transversal intersection of the image of a local embedding $h$ of $Q_{c}^{n+1}$ into $Q_{c}^{n+p+1}$ with $Q_{\hat{c}}^{n+p}$ contained in $Q_{c}^{n+p+1}$ as an umbilical hypersurface. The umbilical foliation is the intersection with $Q_{\tilde{c}}^{n+p}$ of the leaves of the relative nullity foliation of $h$ and has at least dimension $n-s_{g}$. The latter follows from the fact that the leaves of the relative nullity foliation of $h$ have at least dimension $n-s_{h}+1$, and $s_{h}=s_{g}$ from our assumption of transversality. Applying Theorem 2 we can easily provide conditions which imply that the composition is global; namely, if $f$ is an embedding and $g$ is 1-regular and has flat normal bundle.

Using the classical Cartan-Schouten theory for conformally flat hypersurfaces for dimension $n \geq 4$, it followed from the do Carmo-Dajczer result that only conformally flat manifolds can be realized as hypersurfaces of two space forms of different sectional curvature. After extending Theorem 3 to the case where $s_{g}=n-2$, we conclude $\S 2$ by showing that for dimension $n=3$ this is no longer the case.

Classifying Euclidean pseudo-umbilical submanifolds of codimension 2 has been attempted by Yano and Ishihara [20] and Otsuki [17]. Recall that an isometric immersion $g: M^{n} \rightarrow \mathbf{R}^{n+p}$ is said to be pseudo-umbilical if the (normalized) mean curvature vector $H$ is an umbilical vector field. If, in addition, $H$ is parallel in the normal bundle of $g$, then it is easy to show that $g\left(M^{n}\right)$ is contained in an umbilical hypersurface of $\mathbf{R}^{n+p}$ as a minimal submanifold. Among several other related results, the following complete description is given in $\S 3$.

Theorem 4. Any pseudo-umbilical isometric immersion $f: M^{n} \rightarrow \mathbf{R}^{n+2}$, $n \geq 3$, without umbilical points and nonparallel mean curvature vector is a composition $g=h \circ f$. Here $f: M^{n} \rightarrow \mathbf{R}^{n+1}$ is a rotation hypersurface with axis $e_{n+1}$ and principal curvatures $\gamma, \beta>0$ of multiplicities 1 and $n-1$, respectively, satisfying $-(n-1) \beta<\gamma<\beta$, and the map $h=\operatorname{Id} \times c: \mathbf{R}^{n} \times \mathbf{R} \rightarrow \mathbf{R}^{n+2}$ is the right cylinder over the unit speed plane curve $c=c(s)$ so that

$$
(\gamma-\beta)(\gamma+(n-1) \beta)+(k \cos \theta)^{2}=0,
$$

where $k(s)$ is the curvature of $c(s)$ and $\theta(s)$ is the angle between $e_{n+1}$ and the principal direction correspondent to $\gamma$.

This paper was completed while the authors were on a leave of absence at SUNY at Stony Brook. It is a pleasure to acknowledge the hospitality and kindness of the people of the Stony Brook Mathematics Department. 


\section{Isometric compositions}

We first fix some notation. Let $f: M^{n} \rightarrow Q_{c}^{n+p}$ be an isometric immersion with vector-valued second fundamental form $\alpha_{f}: T M \times T M \rightarrow$ $T M^{\perp}$. We denote by $\nabla^{\perp}$ the connection induced on $T M^{\perp}$ by the connection $\widetilde{\nabla}$ of the ambient space. For a normal vector $\xi \in T_{x} M^{\perp}$, we denote by $A_{\xi}^{f}$ the associated endomorphism of $T_{x} M$ defined by

$$
\left\langle A_{\xi}^{f} X, Y\right\rangle=\left\langle\alpha_{f}(X, Y), \xi\right\rangle .
$$

Given isometric immersions $f: M^{n} \rightarrow Q_{c}^{n+p}$ and $g: M^{n} \rightarrow Q_{c}^{n+p+q}$, $q \geq 1$, we say that $\alpha_{g}$ decomposes as $\alpha_{g}=\alpha_{f} \oplus \gamma$ if there exists a smooth vector bundle isometry $\tau: T_{f} M^{\perp} \rightarrow L \subset T_{g} M^{\perp}$ such that $\alpha_{g}$ splits orthogonally and smoothly as $\alpha_{g}=\tau \circ \alpha_{f} \oplus \gamma$.

The proofs of Theorem 1 and 2 stated in the Introduction rely heavily on the following result, which actually deals with a more general setting and should be useful in other situations.

Theorem 5. Let $f: M^{n} \rightarrow Q_{c}^{n+p}$ be an isometric embedding and let $g: M^{n} \rightarrow Q_{c}^{n+p+q}, q \geq 1$, be an isometric immersion whose second fundamental form decomposes as $\alpha_{g}=\alpha_{f} \oplus \gamma$. Assume further that $\tau$ is parallel with respect to the induced connection on $L$, and that there exists a smooth rank-p vector subbundle $\Gamma \subset T M \oplus L$ with $\Gamma \cap T M=\{0\}$ so that

$$
\widetilde{\nabla}_{Z} \mu \in T M \oplus L
$$

for all $\mu \in \Gamma$ and $Z \in T M$. Then $g$ is a composition.

Proof. We consider the case $c=0$, since the proof of the other cases is similar. Consider the vector bundle isometry

$$
T=\operatorname{Id} \oplus \tau: T M \oplus T_{f} M^{\perp} \rightarrow T M \oplus L,
$$

where Id is the identity endomorphism in $T M$, and set $\Omega=T^{-1}(\Gamma)$. By assumption, $\Omega$ is transversal to $T M$. Thus the map $F: \Omega \rightarrow \mathbf{R}^{n+p}$ defined by

$$
F(\xi(x))=f(x)+\xi(x)
$$

parametrizes a tubular neighborhood of $f(M)$ if it is restricted to a neighborhood $U$ of the 0 -section of $\Omega$.

We claim that the map $G: \Omega \rightarrow \mathbf{R}^{n+p+q}$ given by

$$
G(\xi(x))=g(x)+T \xi(x)
$$

is isometric on $U$ with respect to the flat metric induced by $F$. Given a local section $\xi \in \Omega$, set $\xi=X+\delta$, where $X \in T M$ and $\delta \in T_{f} M^{\perp}$. 
Using the assumption on $\Gamma$, we have

$$
\begin{aligned}
G_{*}(\xi(x)) Z & =g_{*}(x) Z+\tilde{\nabla}_{Z} T(X+\delta) \\
& =g_{*}(x)\left(Z+\nabla_{Z} X-A_{\tau(\delta)}^{g} Z\right)+\left(\alpha_{g}(X, Z)+\nabla_{Z}^{g \perp} \tau(\delta)\right)_{L} \\
& =(g \circ f)_{*}^{-1}\left(f_{*}(x)\left(Z-\nabla_{Z} X-A_{\delta}^{f} Z\right)\right)+\tau\left(\alpha_{f}(X, Z)+\nabla_{Z}^{f \perp} \delta\right),
\end{aligned}
$$

and now the claim follows easily. We conclude that the map

$$
h=\left(\left.G\right|_{U}\right) \circ\left(\left.F\right|_{U}\right)^{-1}: F(U) \rightarrow \mathbf{R}^{n+p+q}
$$

is an isometric immersion and $g=h \circ f$. This ends the proof.

Remark 1. Following Henke [12] we call $h$ an isometric extension of $f$. Here $h$ has been constructed to have the orthogonal complement of $L$ in $T_{g} M^{\perp}$ as its normal bundle, and the fibers of $\Gamma$ as part of its relative nullity foliation. It is easy to see that under these conditions the isometric extension $h$ is unique.

Our next goal is to present several conditions on $f: M^{n} \rightarrow Q_{c}^{n+1}$ and $g: M^{n} \rightarrow Q_{c}^{n+p}$ which will allow us to conclude, by means of Theorem 5, that $g$ is a global composition. For that we will make use of various facts and consequences from the theory of flat bilinear forms which, in fact, will be crucial throughout the whole paper.

Let $V$ and $W$ be finite-dimensional real vector spaces and $\beta: V \times V \rightarrow$ $W$ a bilinear map. We denote by $N(\beta)$ the nullity space of $\beta$ defined by

$$
N(\beta)=\{n \in V: \beta(X, n)=0 \forall X \in V\},
$$

and by the image of $\beta$ the subspace $S(\beta)$ of $W$ spanned by $\beta$. For an element $X \in V, \beta(X): V \rightarrow W$ will represent the linear map defined by $\beta(X)(Y)=\beta(X, Y)$. Our interest lies on those $\beta$ which are flat with respect to a nondegenerate bilinear form (inner product) $\langle\rangle:, W \times W \rightarrow$ $\mathbf{R}$, that is,

$$
\langle\beta(X, Y), \beta(Z, W)\rangle-\langle\beta(X, W), \beta(Z, Y)\rangle=0
$$

for all $X, Y, Z, W \in V$. In the following result we put together all we need about flat bilinear forms.

Proposition 6. Let $\beta: V \times V \rightarrow W$ be a flat bilinear form with $S(\beta)=$ $W$, where $\langle$, > has either positive definite or Lorentzian signature. Then

$$
\operatorname{dim} N(\beta) \geq \operatorname{dim} V-\operatorname{dim} W
$$

and, if equality holds, there exists an element $X \in V$ so that $\beta(X)$ is onto. If, in addition, equality holds, $\beta$ is symmetric, and in the Lorentzian case 
there exists $e \in W$ so that $\langle\beta, e\rangle$ is positive definite, then $\beta$ decomposes as

$$
\beta=\beta_{1} \oplus \cdots \oplus \beta_{l}, \quad l=\operatorname{dim} W,
$$

where $S\left(\beta_{i}\right)=W_{i}$ is one-dimensional and nondegenerate, $W_{i} \perp W_{j}$ if $i \neq j$, and each $\beta_{i}$ is flat. Moreover, the subspaces $W_{i}$ are uniquely determined up to permutations.

Proof. Except for the last assertion, the theorem is completely proven in [14]. To show uniqueness we first sketch Moore's argument which provides the decomposition.

Without loss of generality we may assume that $N(\beta)=\{0\}$ and $\operatorname{dim} V$ $=\operatorname{dim} W$. Let $X \in V$ be such that $\beta(X)$ is onto. For each $Y \in V$ define a linear endomorphism $B(Y)$ of $W$ by setting

$$
B(Y)=\beta(Y) \circ \beta(X)^{-1} \text {. }
$$

It is easy to see using flatness of $\beta$ that the $B(Y)$ 's form a commutative Lie algebra of endomorphisms of $W$ which are symmetric with respect to $\langle$,$\rangle . If \langle$,$\rangle is positive definite, the B(Y)$ 's can be simultaneously diagonalized by an orthonormal bases $\xi_{1}, \cdots, \xi_{l}$ of $W$. It is shown in [14] that this is also the case when $\langle$,$\rangle has Lorentzian signature and the$ additional hypothesis is assumed. Let $W_{i}=\operatorname{span}\left\{\xi_{i}\right\}$ and let $\beta_{i}$ be the $W_{i}$-component of $\beta$. Then there are linear functionals $\mu_{i}$ on $V$ such that

$$
\left.B(Y)\right|_{W_{i}}=\mu_{i}(Y) \mathbf{I d},
$$

where Id is the identity endomorphism. Therefore,

$$
\beta_{i}(Y,)=\mu_{i}(Y) \beta_{i}(X,) \text {. }
$$

It follows that each $\beta_{i}$ is flat and $\beta=\beta_{1} \oplus \cdots \oplus \beta_{l}$.

To prove uniqueness of the $W_{i}$ 's it suffices to show the existence of an element $Y_{0} \in V$ for which $\mu_{i}\left(Y_{0}\right) \neq \mu_{j}\left(Y_{0}\right)$ whenever $i \neq j$, since in this case the $W_{i}$ 's will be the one-dimensional eigenspaces associated to the distinct eigenvalues of $B\left(Y_{0}\right)$.

If $\mu_{i} \neq \mu_{j}$ for $i \neq j$, then we may simply choose any element $Y_{0} \notin$ $\bigcup_{i, j} \operatorname{Ker}\left(\mu_{i}-\mu_{j}\right)$. So it remains to show that $\mu_{i}=\mu_{j}$ for $i \neq j$ leads to a contradiction. Let $\xi_{i}=\beta(X)\left(Z_{i}\right)$ and $\xi_{j}=\beta(X)\left(Z_{j}\right)$. Using flatness, we have

$$
\begin{aligned}
\mu_{i}(Y) & =\left\langle B(Y) \xi_{i}, \xi_{i}\right\rangle=\left\langle\beta(Y) \beta(X)^{-1} \xi_{i}, \xi_{i}\right\rangle \\
& =\left\langle\beta(Y) Z_{i}, \beta(X) Z_{i}\right\rangle=\left\langle\beta\left(Y, Z_{i}\right), \beta\left(X, Z_{i}\right)\right\rangle \\
& =\left\langle\beta(X, Y), \beta\left(Z_{i}, Z_{i}\right)\right\rangle .
\end{aligned}
$$


Therefore,

$$
\left(\mu_{i}-\mu_{j}\right)(Y)=\left\langle\beta(X)(Y), \beta\left(Z_{i}, Z_{i}\right)-\beta\left(Z_{j}, Z_{j}\right)\right\rangle=0 .
$$

Thus, $\beta\left(Z_{i}, Z_{i}\right)=\beta\left(Z_{j}, Z_{j}\right)$ since $\beta(X)$ is onto. But $\beta\left(Z_{i}, Z_{i}\right)=$ $\beta\left(Z_{i}\right) \beta(X)^{-1} \xi_{i}=B\left(Z_{i}\right) \xi_{i}=\mu_{i}\left(Z_{i}\right) \xi_{i}$, and analogously, $\beta\left(Z_{j}, Z_{j}\right)=$ $\mu_{j}\left(Z_{j}\right) \xi_{j}$. We conclude that $\beta\left(Z_{i}, Z_{i}\right)=\beta\left(Z_{j}, Z_{j}\right)=0$, which is in contradiction with

$$
\left\langle\beta\left(Z_{i}, Z_{i}\right), \beta(X, X)\right\rangle=\left\langle\beta(X) Z_{i}, \beta(X) Z_{i}\right\rangle=\left\langle\xi_{i}, \xi_{i}\right\rangle=1,
$$

and concludes the proof.

Let $(E, \pi, M)$ denote a pseudo-Riemannian vector bundle of rank $s$ over a differentiable $n$-dimensional manifold $M$. As usual, we refer to $(E, \pi, M)$ simply by $E$. The vector bundle over $M$ whose fiber at $x \in M$ is the vector space of all $r$-linear symmetric maps $T_{x} M \times \therefore \times$ $T_{x} M \rightarrow E_{x}$ will be denoted by $S^{r}(M ; E)$. For $E=M \times \mathbf{R}$ we simply write $S^{r}(M)$. We denote by $\Gamma(E)$ (respectively, $\Gamma\left(S^{r}(M ; E)\right)$ ) the set of all sections of $E$ (respectively, $S^{r}(M ; E)$ ), and by $C^{\infty}(E)$ (respectively, $\left.C^{\infty}\left(S^{r}(M ; E)\right)\right)$ the set of all smooth sections.

Proposition 7. Let $(E, \pi, M)$ be either Riemannian or Lorentzian of rank $s$, and let $\beta \in C^{\infty}\left(S^{2}(M ; E)\right)$ so that $S(\beta(x))=E_{x}$ for all $x \in M$. Assume further that for every $x \in M, \beta(x)$ is flat, $\operatorname{dim} N(\beta(x))=n-s$, and in the Lorentzian case there exists $e \in \Gamma(E)$ so that $\langle\beta(x), e\rangle$ is positive definite. If $M$ is simply connected, then there exist orthonormal $\xi_{1}, \cdots, \xi_{s} \in C^{\infty}(E)$ so that each $\left\langle\beta, \xi_{j}\right\rangle$ has everywhere rank one.

Proof. It is sufficient to consider in the proof of Proposition 6 the distinct eigenvectors of $B(Y)$, where $Y$ is a local smooth extension of $Y_{0}$. The rank conclusion is equivalent to flatness. q.e.d.

Recall that a submanifold is $k$-ruled if it admits a continuous $k$-dimensional foliation by totally geodesic submanifolds of the ambient space. We will make use of the following result.

Theorem 8. Let $f: M^{n} \rightarrow Q_{c}^{n+1}$ be a nontotally geodesic isometric embedding and let $g: M^{n} \rightarrow Q_{c}^{n+p}$ be a 1-regular isometric immersion so that $\alpha_{g}$ decomposes as $\alpha_{g}=\alpha_{f} \oplus \gamma$ with $\operatorname{dim} N(\gamma(x))=k$ a positive constant. Assume also that either

(i) $N_{1}^{g}$ is parallel

or one of the following conditions holds everywhere:

(ii) $\rho_{f}(x) \geq n-k+2$,

(iii) $\rho_{f}(x) \geq s_{g}+1$,

(iv) $k \geq 2$ and the index of relative nullity $\nu_{g}(x)=\operatorname{dim} N\left(\alpha_{g}(x)\right) \leq$ $k-2$. 
If $s_{g} \geq 4$ and $k \geq n-s_{g}+2$, suppose further that $M^{n}$ does not contain an open $k$-ruled subset for both $f$ and $g$. Then $g$ is a composition.

Proof. For all $x \in M$, we have from the Gauss equations for $f$ and $g$ that $\gamma(x)$ is flat. Proposition 6 yields

$$
k \geq n-\operatorname{dim} S(\gamma(x))=n-s_{g}+1 .
$$

From our assumptions, $L=S\left(\alpha_{f}\right)$ is a globally defined line bundle. Let $\eta$ be a local section of $L$. By comparing Codazzi's equations of $f$ and $g$ for $A_{\eta}$, we get

$$
A_{\nabla_{X}^{\perp} \eta} Z=A_{\nabla_{Z}^{\frac{1}{\eta}} \eta} X
$$

for all $X, Z \in T M$. It follows that

$$
\left(\nabla_{Y}^{\perp} \eta\right)_{N_{1}^{g}}=0 \text { for all } Y \in N(\gamma)
$$

We claim that

$$
\nabla_{X}^{\perp} \eta \in N_{1}^{g} \quad \text { for all } X \in T M
$$

which is trivial if we assume (i). For the remaining cases, suppose there exists a normal vector field $\delta \in N_{1}^{g \perp}$ such that $\left\langle\nabla_{X_{0}}^{\perp} \eta, \delta\right\rangle \neq 0$ for some $X_{0} \in T_{x} M$. From the Codazzi equation for $A_{\delta}(=0)$, we have that

$$
A_{\nabla_{X}^{1} \delta} Y=A_{\nabla_{Y}^{\perp} \delta} X
$$

from which we conclude that

$$
\left\langle\nabla_{X}^{\perp} \delta, \eta\right\rangle A_{\eta} Y-\left\langle\nabla_{Y}^{\perp} \delta, \eta\right\rangle A_{\eta} X \in N(\gamma)^{\perp}
$$

for all $X, Y \in T M$. Now (6) and our assumption yield

$$
A_{\eta} Z \in N(\gamma(x))^{\perp}
$$

for any $Z$ in the $(n-1)$-dimensional kernel of the linear functional $Z \mapsto$ $\left\langle\nabla_{Z}^{\perp} \eta, \delta\right\rangle$. This easily implies

$$
\rho_{f}(x) \leq \operatorname{dim} N(\gamma(x))^{\perp}+1=n-k+1
$$

and

$$
\nu_{g}(x) \geq k-1,
$$

which is in contradiction with either (ii), (iii) using (2) or (iv), and proves the claim.

From Codazzi's equation for $\xi \in T M^{\perp} \cap L^{\perp}, Y \in N(\gamma)$, and any $X \in T M$, we have

$$
\nabla_{Y} A_{\xi} X+A_{\nabla_{X}^{\perp} \xi} Y+A_{\xi}[X, Y]-A_{\nabla_{Y}^{\frac{1}{\xi}}} X=0 .
$$


By taking the inner product of (7) with $Z \in N(\gamma)$ and using (4) and (5), we get

which is equivalent to

$$
\left\langle\nabla_{Y} A_{\xi} X, Z\right\rangle+\left\langle\nabla_{X}^{\perp} \xi, \eta\right\rangle\left\langle\alpha_{g}(Y, Z), \eta\right\rangle=0,
$$

$$
\left\langle\widetilde{\nabla}_{Y} Z,-A_{\xi} X+\left\langle\nabla_{X}^{\perp} \xi, \eta\right\rangle \eta\right\rangle=0 .
$$

Assume that $M^{n}$ is not $k$-ruled. We claim that the subspaces $W(x) \subset$ $N(\gamma(x))^{\perp} \oplus L(x)$ defined by

$$
W(x)=\operatorname{span}\left\{\left(\tilde{\nabla}_{X} \xi\right)_{T M \oplus L}: X \in T_{x} M, \xi \in T M^{\perp} \cap L^{\perp}\right\}
$$

form a one-codimensional subbundle of $N(\gamma)^{\perp} \oplus L$. Clearly, the claim is equivalent to $L$ being everywhere transversal to $W$. If $W(y)=N(\gamma(y))^{\perp}$ $\oplus L(y)$ at some point $y \in M$, then the same holds in a neighborhood $V$. It follows from $(8)$ that on $V$

$$
\left\langle\tilde{\nabla}_{Y} Z, \eta\right\rangle=\left\langle A_{\eta} Y, Z\right\rangle=0 \text { and }\left\langle\nabla_{Y} Z, X\right\rangle=0
$$

for all $Y, Z \in N(\gamma), X \in N(\gamma)^{\perp}$. Thus $N(\gamma)$ is integrable and the leaves are totally geodesic in $Q_{c}^{n+1}$ and $Q_{c}^{n+p}$. This contradicts the assumption of the theorem and proves the claim.

To obtain the proof from Theorem 5 , it suffices to take $\Gamma$ there to be the orthogonal complement of $W$ in $N(\gamma)^{\perp} \oplus L$. If $k \geq n-s_{g}+2$, from $s_{g}=1+\operatorname{dim} S(\gamma) \leq 1+\frac{1}{2}(n-k)(n-k+1)$ we have that $s_{g} \geq 4$. So, to conclude the proof, it remains to show that our hypothesis that $M$ does not contain an open $k$-ruled submanifold can be dropped if $\operatorname{dim} N(\gamma(x))$ takes everywhere its minimum value $n-s_{g}+1$. But if this is the case, Proposition 7 implies that $\alpha_{g}$ splits orthogonally and smoothly as a direct sum,

$$
\alpha_{g}=\alpha_{f} \oplus \gamma_{1} \oplus \cdots \oplus \gamma_{s_{g}-1}
$$

with rank $\gamma_{j}=1,1 \leq j \leq s_{g}-1$. Consider an orthonormal frame $\xi_{1}, \cdots, \xi_{s_{g}-1}$ so that $S\left(\gamma_{j}\right)=\operatorname{span}\left\{\xi_{j}\right\}$. Let $X_{1}, \cdots, X_{s_{g}-1}$ be local unit tangent vector fields so that $X_{j}$ is the eigenvector of $A_{\xi_{j}}$ correspondent to the unique nonzero eigenvalue $\lambda_{j}$. By assumption, $X_{1}, \cdots, X_{s_{g}-1}$ are linearly independent. (3) yields

$$
\sum_{k=1}^{s_{g}-1} \lambda_{k}\left[\left\langle\nabla_{X_{i}}^{\perp} \eta, \xi_{k}\right\rangle\left\langle X_{j}, X_{k}\right\rangle-\left\langle\nabla_{X_{j}}^{\perp} \eta, \xi_{k}\right\rangle\left\langle X_{i}, X_{k}\right\rangle\right] X_{k}=0,
$$

which implies that

$$
\left\langle\nabla_{X_{i}}^{\perp} \eta, \xi_{k}\right\rangle=\left\langle\nabla_{X_{k}}^{\perp} \eta, \xi_{k}\right\rangle\left\langle X_{i}, X_{k}\right\rangle
$$


Therefore,

$$
\left\langle\nabla_{Z}^{\perp} \xi_{k}, \eta\right\rangle=0 \text { for all } Z \perp X_{k}
$$

From (9), we have

$$
W=\operatorname{span}\left\{-A_{\xi_{j}} X_{j}+\left\langle\nabla_{X_{j}}^{\perp} \xi_{j}, \eta\right\rangle \eta: 1 \leq j \leq s_{g}-1\right\}
$$

which again implies that $W$ has codimension one in $N(\gamma)^{\perp} \oplus L$. This concludes the proof.

Remarks 2. (i) If the sectional curvature of $M$ satisfies $K_{M}>c$, then $f$ cannot be ruled.

(ii) The isometric extension $h$ of $f$ has index of relative nullity $\nu_{h}=$ $k+1$ and is unique if $k=n-s_{g}+1$ (cf. Remark 1).

(iii) If $g$ has flat normal bundle, then $k=n-s_{g}+1$. In fact, let $X_{1}, \cdots, X_{n}$ be an orthonormal basis diagonalizing $\alpha_{g}$ so that $N(\gamma(x))^{\perp}$ $=\operatorname{span}\left\{X_{1}, \cdots, X_{n-k}\right\}$. Then

$$
S(\gamma(x))=\operatorname{span}\left\{\gamma\left(X_{i}, X_{i}\right): 1 \leq i \leq n-k\right\}
$$

and the opposite inequality $k \leq n-s_{g}+1$ also holds.

For the proof of Theorem 2 we need the following.

Proposition 9. Let $(E, \pi, M)$ be a Riemannian vector bundle of rank $s$ and let $\beta \in C^{\infty}\left(S^{2}(M ; E)\right)$ be so that $S(\beta(x))=E_{x}$ for all $x \in M$. Assume further that $\beta$ splits orthogonally as $\beta=\varphi \eta \oplus \gamma$, where $\varphi \in$ $C^{\infty}\left(S^{2}(M)\right), \eta \in \Gamma(E)$ with $\|\eta\|=1$, and $\gamma \in \Gamma\left(S^{2}(M ; E)\right)$. If $\varphi$ never vanishes, then $\eta \in C^{\infty}(E)$ and $\gamma \in C^{\infty}\left(S^{2}(M ; E)\right)$.

Proof. At $x \in M$, let $\left(X_{i_{k}}, X_{j_{k}}\right), 1 \leq k \leq s$, be a set of pairs so that

$$
E_{x}=\operatorname{span}\left\{\beta\left(X_{i_{k}}, X_{j_{k}}\right): 1 \leq k \leq s\right\}
$$

Extending the pairs $\left(X_{i_{k}}, X_{j_{k}}\right)$ smoothly in a neighborhood of $x$, one still has that the $\beta\left(X_{i_{k}}, X_{j_{k}}\right)$ 's form a basis of $E_{y}$ for any $y$ in a neighborhood $U$ of $x$. In particular, there are well defined functions $f_{k}, 1 \leq k \leq s$, in $U$ such that

$$
\eta=\sum_{k=1}^{s} f_{k} \beta\left(X_{i_{k}}, X_{j_{k}}\right) .
$$

On the other hand, the functions $\varphi_{k}=\varphi\left(X_{i_{k}}, X_{j_{k}}\right), 1 \leq k \leq s$, are smooth and cannot all vanish simultaneously at any point of $U$. Moreover

$$
\varphi_{r}=\left\langle\beta\left(X_{i_{r}}, X_{j_{r}}\right), \eta\right\rangle=\sum_{k} f_{k}\left\langle\beta\left(X_{i_{r}}, X_{j_{r}}\right), \beta\left(X_{i_{k}}, X_{j_{k}}\right)\right\rangle=\sum_{k} f_{k} \psi_{r k}
$$


The functions $\psi_{r k}, 1 \leq r, k \leq s$, are smooth and the matrix $\left(\psi_{r k}\right)$ is invertible at any point in $U$. We conclude that the $f_{k}$ 's are smooth as we wished.

Proof of Theorem 2. At $x \in M$, define a Lorentzian inner product on $W=T_{x}^{f} M^{\perp} \oplus N_{1}^{g}(x)$ by

$$
\left\langle\left\langle\left(\xi_{1}, \xi_{2}\right),\left(\delta_{1}, \delta_{2}\right)\right\rangle\right\rangle=-\left\langle\xi_{1}, \delta_{1}\right\rangle_{T^{f} M^{\perp}}+\left\langle\xi_{2}, \delta_{2}\right\rangle_{T^{g} M^{\perp}}
$$

By the Gauss equations for $f$ and $g$, the symmetric bilinear form $\beta: T_{x} M$ $\times T_{x} M \rightarrow W$ defined by

$$
\beta(X, Y)=\left(\alpha_{f}(X, Y), \alpha_{g}(X, Y)\right)
$$

is flat with respect to $\langle\langle\rangle$,$\rangle . Thus \operatorname{dim} N(\beta) \leq n-\rho_{f}(x)$, which forces $S(\beta)$ to be degenerate. Otherwise, we would have from our hypothesis and Proposition 6 that

$$
\operatorname{dim} N(\beta) \geq n-\operatorname{dim} S(\beta) \geq n-s_{g}-1 \geq n-\rho_{f}(x)+1,
$$

a contradiction. Hence, there exists a unit vector $\eta \in N_{1}^{g}(x)$ so that

$$
(N, \eta) \in S(\beta) \cap S(\beta)^{\perp},
$$

where $N$ is a unit vector normal to $f$ at $x$. This is equivalent to

$$
\left\langle\alpha_{f}(X, Y), N\right\rangle_{T^{f} M^{\perp}}=\left\langle\alpha_{g}(X, Y), \eta\right\rangle_{T^{g} M^{\perp}}
$$

Thus, we can write

$$
\alpha_{g}(x)=\left\langle A_{N},\right\rangle \eta \oplus \gamma(x),
$$

where $\gamma(x)$, the $\{\eta\}^{\perp}$-component of $\alpha_{g}(x)$, is flat. In particular,

$$
\operatorname{dim} N(\gamma(x)) \geq n-\operatorname{dim} S(\gamma(x))=n-s_{g}+1 .
$$

Part (a) of the theorem now follows from Proposition 9.

To conclude the proof of part (b) from Theorem 8, first observe that, in general, if $f$ is $l$-ruled, then $\rho_{f} \leq 2(n-l)$. Therefore, if $f$ is $\left(n-s_{g}+2\right)$ ruled, we have

$$
s_{g}+2 \leq \rho_{f} \leq 2\left(s_{g}-2\right)
$$

which can only occur if $s_{g} \geq 6$.

Remarks 3. (i) Part (b) of Theorem 2 may not hold if $f$ is only an immersion instead of an embedding (cf. [5]). But it is still true from the proof that there exist a manifold $N_{c}^{n+1}$ of constant sectional curvature $c$ and isometric immersions $f^{\prime}: M^{n} \rightarrow N_{c}^{n+1}$ and $h: N_{c}^{n+1} \rightarrow Q_{c}^{n+p}$ such that $f^{\prime}$ is locally congruent to $f$ and $g=h \circ f^{\prime}$. 
(ii) No additional assumption is needed for $s_{g} \geq 6$ if the sectional curvature of $M$ satisfies $K_{M} \geq c$. Under this condition, the existence of $k$-dimensional rulings with $k \geq n-s_{g}+2$ would imply that $\rho_{f}<s_{g}+2$.

(iii) For $p=2$, Theorem 2 was already obtained in [5].

We conclude this section by extending to convex hypersurfaces a result proved by O'Neill [16] for the round sphere.

Theorem 10. Let $f: M^{n} \rightarrow \mathbf{R}^{n+1}, n \geq 4$, be an isometric immersion of a compact manifold of positive sectional curvature. Then there is no 1-regular isometric immersion of $M^{n}$ into $\mathbf{R}^{n+2}$ other than the trivial one.

Proof. Assume that $g: M^{n} \rightarrow \mathbf{R}^{n+2}$ is a 1-regular isometric immersion, and let $h: U \subset \mathbf{R}^{n+1} \rightarrow \mathbf{R}^{n+2}$ be the isometric extension of $f$ given by Theorem 2. Suppose $s_{g} \equiv 2$. Then $\rho_{h}=1$ everywhere, and the $n$-dimensional leaves of the relative nullity distribution of $h$ are transversal to $f(M)$. Hence, their intersections with $f(M)$ provide a one-codimensional foliation of $M$ whose leaves are easily seen to be complete. This is clearly a contradiction. Thus, $s_{g} \equiv 1$ and $h$ is just an inclusion.

\section{Submanifolds of two manifolds}

First we prove Theorem 3 from the Introduction.

Proof of Theorem 3. To reduce the proof to Theorem 8, we start by showing that the second fundamental form of $G$ decomposes as $\alpha_{G}=$ $\alpha_{f} \oplus \gamma$. This follows from the proof of Theorem 2 if we show that the flat symmetric bilinear form $\beta=\left(\alpha_{f}, \alpha_{G}\right)$ satisfies $N(\beta)=\{0\}$. To see this, let $\delta$ denote a unit normal vector field to the inclusion of $Q_{\hat{c}}^{n+p}$ into $Q_{c}^{n+p+1}$. Then

$$
\left\langle\alpha_{G}, \delta\right\rangle=\sqrt{\tilde{c}-c}\langle,\rangle,
$$

and in particular, $\nu_{G}=0$ everywhere. Thus $N(\beta)=0$.

It also follows from (10) that $G$ cannot be ruled. To conclude the proof, observe that from $s_{g} \leq n-3$ we have $\operatorname{dim} N(\gamma(x)) \geq n-s_{g} \geq 3$, so $G$ satisfies condition (iv) of Theorem 8 . This concludes the proof.

Next we extend Theorem 3 to the case $s_{g}=n-2$.

Theorem 11. The same conclusion as in Theorem 3 holds if $s_{g}(x)=$ $n-2$ everywhere, unless there exists an open subset of $M$ where the second fundamental forms $\alpha_{f}$ and $\alpha_{g}$ can be simultaneously orthogonally diagonalized. 
Proof. Let $\beta$ and $\delta$ be as in the proof of Theorem 3. It is sufficient to show that $S(\beta)$ is a degenerate subspace everywhere. If $S(\beta(x))$ is nondegenerate at $x \in M$, then the same holds in some open neighborhood $V$ of $x$. From Proposition 6 and $N(\beta(y))=\{0\}$, we have that $\operatorname{dim} S(\beta(y))=n$ for all $y \in V$. It follows from Propositions 6 and 7 (Lorentzian case) that there exist a smooth orthonormal basis $\xi_{1}, \cdots, \xi_{n}$ of $T^{f} M^{\perp} \oplus N_{1}^{g}$ and a basis $\theta^{1}, \cdots, \theta^{n}$ of $T M^{*}$ so that

$$
\beta=\sum_{j=1}^{n} \theta^{j} \otimes \theta^{j} \xi_{j} .
$$

Thus, $\beta\left(Z_{i}, Z_{j}\right)=0$ for $i \neq j$, where $Z_{1}, \cdots, Z_{n}$ is the dual basis to $\theta^{1}, \cdots, \theta^{n}$. On the other hand,

$$
0=\left\langle\left\langle\beta\left(Z_{i}, Z_{j}\right), \delta\right\rangle\right\rangle=\sqrt{\tilde{c}-c}\left(Z_{i}, Z_{j}\right\rangle,
$$

that is, $Z_{1}, \cdots, Z_{n}$ is an orthogonal basis. This completes the proof.

Assume that the Riemannian manifold $M^{3}$ admits isometric immersions $f: M^{3} \rightarrow Q_{c}^{4}$ and $g: M^{3} \rightarrow Q_{\tilde{c}}^{4}$ with $c<\tilde{c}$, so that $G=i \circ g: M^{3} \rightarrow$ $Q_{c}^{5}$ is nowhere a composition. By Theorem 11, the second fundamental forms of $f$ and $g$ can be simultaneously orthogonally diagonalized at any point of $M$. Using this fact, a long but straightforward computation yields the following result.

Proposition 12. Let $f: M^{3} \rightarrow Q_{c}^{4}$ be an isometric immersion of a simply connected Riemannian manifold with principal directions $e_{1}, e_{2}, e_{3}$, and correspondent distinct principal curvatures $\lambda_{1}, \lambda_{2}, \lambda_{3}$. Then $M^{3}$ can be isometrically immersed into $Q_{\tilde{c}}^{4}, c \neq \tilde{c}$, if and only if for all indices $i \neq j \neq k$

$$
\begin{gathered}
\left\langle\nabla_{e_{i}} e_{j}, e_{k}\right\rangle=0 \\
\left(\lambda_{i} \lambda_{k}-1\right)\left(\lambda_{k}-\lambda_{i}\right) e_{i}\left(\lambda_{j}\right)+\left(\lambda_{j} \lambda_{i}-1\right)\left(\lambda_{i}-\lambda_{j}\right) e_{i}\left(\lambda_{k}\right) \\
+\left(\lambda_{j} \lambda_{k}-1\right)\left(\lambda_{k}-\lambda_{j}\right) e_{i}\left(\lambda_{i}\right)=0
\end{gathered}
$$

Proposition 12 provides a negative answer to the question of whether $M^{3}$ must be conformally flat. In fact, conformally flat hypersurfaces $M^{3}$ of $Q_{c}^{4}$ have been characterized by E. Cartan (see [13, p. 84]) by the following relations:

$$
\begin{gathered}
\left\langle\nabla_{e_{i}} e_{j}, e_{k}\right\rangle=0 \\
\left(\lambda_{j}-\lambda_{k}\right) e_{i}\left(\lambda_{i}\right)+\left(\lambda_{i}-\lambda_{k}\right) e_{i}\left(\lambda_{j}\right)+\left(\lambda_{j}-\lambda_{i}\right) e_{i}\left(\lambda_{k}\right)=0
\end{gathered}
$$

for all indices $i \neq j \neq k$. It is not difficult to show that the second condition of (11) and (12) are both simultaneously satisfied if and only if 
$e_{i}\left(\lambda_{i} \lambda_{j}\right)=0$ for all $i \neq j$. An example of this situation is provided by a cone in $\mathbf{R}^{4}$ over a nonumbilical surface of constant curvature in $S^{3}$ (see $[13$, p. 81$])$.

\section{Further results}

We say that a unit normal vector field $\eta$ to an isometric immersion $g: M^{n} \rightarrow Q_{c}^{n+p}$ is an umbilical vector field if there exists a nowhere vanishing function $\lambda \in C^{\infty}(M)$ so that $A_{\eta}^{g}=\lambda$ Id. It is an elementary fact that $\eta$ is parallel in the normal connection of $g$ if and only if $g(M)$ is contained in an umbilical hypersurface of $Q_{c}^{n+p}$. Our next results shows that for low codimension, umbilical vector fields are "generically" parallel. But first, we recall from [9] the concept of s-nullity, $\nu_{s}(x)$, of $g$ at $x \in M$. By definition,

$$
\nu_{s}(x)=\max _{U^{s} \subset T_{x} M^{\perp}}\left\{\operatorname{dim} N\left(\pi_{U^{s}} \circ \alpha_{g}\right)\right\}, \quad 1 \leq s \leq p,
$$

where $U^{s}$ ranges over all $s$-dimensional vector subspaces of $T_{x} M^{\perp}$, and $\pi_{U^{s}}: T_{x} M^{\perp} \rightarrow U^{s}$ denotes the orthogonal projection onto $U^{s}$.

Proposition 13. Let $g: M^{n} \rightarrow Q_{c}^{n+p}, 2 \leq p \leq n-1$, be an isometric immersion with an umbilical vector field. Assume $\nu_{s}(x)<n-s$ for all $1 \leq s \leq p-1$ and all $x \in M$. Then $g(M)$ is contained in an umbilical hypersurface of $Q_{c}^{n+p}$.

Proof. At $x \in M$, consider the linear map $\varphi: T_{x} M \rightarrow T_{x} M^{\perp} \cap\{\eta\}^{\perp}$ defined by

$$
\varphi(X)=\nabla_{X}^{\perp} \eta,
$$

where $\eta$ is the umbilical vector field. We argue that $\varphi=0$.

Suppose that $\operatorname{dim} \operatorname{Im} \varphi=r>0$. Since $r \leq p-1$, we have $\operatorname{dim} \operatorname{ker} \varphi=$ $n-r \geq 2$. Codazzi's equation for $A_{\eta}$ yields $Y(\lambda) Z=Z(\lambda) Y$ for any $Y, Z \in \operatorname{ker} \varphi$. Thus $Y(\lambda)=0$ for all $Y \in \operatorname{ker} \varphi$. Using Codazzi's equation again, we get for any $Y \in \operatorname{ker} \varphi$ and $X \in T_{x} M$

$$
A_{\psi(x)} Y=0 .
$$

Here $\psi: T_{x} M \rightarrow T_{x} M^{\perp}$ is the linear map defined by

$$
\psi(X)=\varphi(X)-\frac{1}{\lambda} X(\lambda) \eta .
$$

Since $\operatorname{dim} \operatorname{Im} \psi=\operatorname{dim} \operatorname{Im} \varphi=r$, we conclude that $\nu_{r}(x) \geq n-r$, which is a contradiction. Therefore, $r=0$ and this concludes the proof. 
Remark 4. From the proof it is sufficient to assume $\nu_{s}<n-s$ for $1 \leq s \leq \operatorname{dim} \operatorname{Im} \varphi$, and only consider $U^{s} \subset \operatorname{Im} \psi$. This implies Theorem 1 of [4].

Recall that the classical Cartan-Schouten result asserts that any hypersurface $M^{n}$ of $N_{c}^{n+1}, n \geq 3$, which has only principal curvatures of multiplicities $1, n-1$, or $n$ at any point is conformally flat, whereas the converse holds for $n \geq 4$. In particular, if a conformally flat hypersurface has two different principal curvatures everywhere, then it will carry a codimension one foliation by umbilical leaves in both $M^{n}$ and $N_{c}^{n+1}$. (See [10] for a parametric description of such hypersurfaces.)

An umbilical vector field is said to be nonparallel if the associated map $\varphi$ defined in (13) is nonzero everywhere. Isometric immersions $g: M^{n} \rightarrow$ $\mathbf{R}^{n+2}, n \geq 3$, with a nonparallel umbilical vector field have been shown by Chen and Yano [2] to be conformally flat.

Theorem 14. Let $g: M^{n} \rightarrow Q_{c}^{n+2}, n \geq 3$, be an umbilical free isometric immersion with a nonparallel umbilical vector field. Then $g$ is a composition $g=h \circ f$, where $f: M^{n} \rightarrow N_{c}^{n+1}$ is a conformally flat hypersurface and $h: N_{c}^{n+1} \rightarrow Q_{c}^{n+2}$ verifies $\rho_{h}=1$ everywhere. Moreover, on the open subset of nonumbilical points each leaf of the umbilical foliation of $f$ is contained in a leaf of the relative nullity foliation of $h$. Conversely, any such composition has an umbilical vector field.

Proof. Let $\varphi: T M \rightarrow T M^{\perp} \cap\{\eta\}^{\perp}$ be the globally defined linear map given by (13), and let $X$ be a local unit tangent vector field orthogonal to $\operatorname{ker} \varphi$. Using that $g$ has not umbilical points, we obtain from the proof of Proposition 13 that the unit normal vector field

$$
\delta=\frac{1}{\|\psi(x)\|} \psi(x)
$$

satisfies everywhere that $\operatorname{ker} A_{\delta}=\operatorname{ker} \varphi$, and therefore it is globally defined. Thus, for a unit normal vector field $\xi$ orthogonal to $\delta$, we have that $A_{\xi} Y=\beta Y$ for all $Y \in \operatorname{ker} A_{\delta}$. Since $A_{\eta}=\lambda$ Id, where by definition $\lambda$ never vanishes, we conclude that $\xi$ is globally defined by the condition $\beta>0$.

We claim that the endomorphism $A_{\xi}$ satisfies the Gauss and Codazzi equations for an isometric immersion into $Q_{c}^{n+1}$. To prove the claim, first observe that from the Codazzi equation for $A_{\delta}$ we get that $\xi$ is parallel in the normal bundle along $\operatorname{ker} A_{\delta}$. This implies

$$
A_{\nabla \frac{1}{Z} \xi} W=A_{\nabla_{W}^{1} \xi} Z
$$

for all $Z, W \in T M$, and the claim follows easily. The proof of the 
first part of the theorem now follows from Theorem $5^{\prime}$ below, a slightly modified version of Theorem 5 . The converse is left to the reader.

Theorem 5'. Let $g: M^{n} \rightarrow Q_{c}^{n+p+q}$ be an isometric immersion whose normal bundle contains a smooth rank-p subbundle $L \subset T_{g} M^{\perp}$ so that the $L$-valued symmetric bilinear form $\alpha_{L}=\pi_{L} \circ \alpha$ satisfies Gauss, Codazzi, and Ricci equations as a submanifold of $Q_{c}^{n+p}$ with respect to the induced connection on $L$. Assume further that there exists a rank-p subbundle $\Gamma \subset T M \oplus L$ as in Theorem 5. Then there exist a Riemannian manifold $N_{c}^{n+p}$ and isometric immersions $f: M^{n} \rightarrow N_{c}^{n+p}$ and $h: N_{c}^{n+p} \rightarrow Q_{c}^{n+p+q}$ such that $\alpha_{f}=\alpha_{L}$ and $g=h \circ f$.

Remark 5. It is implicit in the statement of Theorem 14 and follows from the proof that $f$ has at most two different principal curvatures even for $n=3$.

We now prove Theorem 4 stated in the Introduction.

Proof of Theorem 4. Let $\delta, \xi$ be the global orthonormal frame constructed in the proof of Theorem 14. Then $A_{\delta}$ has a unique nonzero principal curvature $\mu$, while $A_{\xi}$ possesses two principal curvatures $\gamma, \beta>0$ of multiplicities 1 and $n-1$, respectively. Moreover, $\mu$ and $\gamma$ correspond to the same unit principal direction, which we denote by $X$. Therefore, the mean curvature vector is

$$
H=(\gamma+(n-1) \beta) \xi+\mu \delta,
$$

and from $A_{H}=\lambda$ Id, we easily get

$$
(\gamma-\beta)(\gamma+(n-1) \beta)+\mu^{2}=0
$$

Using Codazzi's equation for $A_{\delta}$, we obtain

$$
\begin{aligned}
& \nabla_{Y}^{\perp} \xi=0, \quad Y(\mu)=\mu\left\langle\nabla_{X} X, Y\right\rangle, \\
& \mu\left\langle\nabla_{Y} Z, X\right\rangle=\beta\left\langle\nabla_{X}^{\perp} \delta, \xi\right\rangle\langle Y, Z\rangle
\end{aligned}
$$

for all $Y, Z \in \operatorname{ker} A_{\delta}$. The Codazzi equation for $A_{\xi}$ and (15) yield for all $Y \in \operatorname{ker} A_{\delta}$

$$
Y(\beta)=0, Y(\gamma)=(\gamma-\beta)\left\langle\nabla_{X} X, Y\right\rangle, \quad\|Y\|^{2} X(\beta)=(\beta-\gamma)\left\langle\nabla_{Y} Y, X\right\rangle .
$$

Since $\mu \neq 0$ we have from (15) and (16) that

$$
\mu Y(\gamma)=(\gamma-\beta) Y(\mu) .
$$

On the other hand, differentiating (14) with respect to $Y$ gives

$$
(2 \gamma+(n-2) \beta) Y(\gamma)+2 \mu Y(\mu)=0 \text {. }
$$


From (17) and (18) it follows that

$$
\left((2 \gamma+(n-2) \beta)(\gamma-\beta)+2 \mu^{2}\right) Y(\gamma)=0 .
$$

Using (14), we get

$$
(\gamma-\beta) \beta Y(\gamma)=0 .
$$

Since $\gamma-\beta \neq 0$ by (14), we conclude that

$$
Y(\gamma)=0 \quad \text { for all } Y \in \operatorname{ker} A_{\delta} .
$$

As in the proof of Theorem 14, $A_{\xi}$ satisfies the Gauss and Codazzi equations for an isometric immersion into $\mathbf{R}^{n+1}$. Thus, there exists an isometric immersion $\tilde{f}: \widetilde{M}^{n} \rightarrow \mathbf{R}^{n+1}$, defined on the universal covering $\widetilde{M}$, whose second fundamental form is $A_{\xi}$. From (19) and Theorem 4.2 of [8], it follows that $f$ is a rotation hypersurface. (14) yields

$$
-(n-1) \beta<\gamma<\beta \text {. }
$$

In particular, $\tilde{f}$ is an embedding. The remainder of the proof is now straightforward.

Remark 6. Chen and Yano [3] also considered pseudo-umbilical isometric immersions $g: M^{n} \rightarrow \mathbf{R}^{n+3}, n \geq 3$, with nonparallel mean curvature vector of constant length and flat normal bundle. It is possible to show that all examples are compositions $g=h \circ f$. Here $f: M^{n} \rightarrow \mathbf{R}^{n+1}$ is a rotation hypersurface with axis $e_{n+1}$ and principal curvatures $\gamma$ and $\beta$ of multiplicities 1 and $n-1$ respectively, isometric to an $n$-catenoid in $S_{c}^{n+1}$ (see [7]), and $h=\operatorname{Id} \times d: \mathbf{R}^{n} \times \mathbf{R} \rightarrow \mathbf{R}^{n+3}$ is a right cylinder over a unit speed plane curve $d=d(s)$ with curvature $k(s)$ satisfying the equation

$$
\gamma(\gamma+(n-1) \beta)+(k \cos \theta)^{2}=n c,
$$

where $\theta(s)$ is the angle between $e_{n+1}$ and the principal direction correspondent to $\gamma$.

\section{References}

[1] A. Asperti \& M. Dajczer, $N$-dimensional submanifolds of $\mathbf{R}^{N+1}$ and $S^{N+2}$, Illinois J. Math. 28 (1984) 621-645.

[2] B. Y. Chen \& K. Yano, Umbilical submanifolds with respect to a nonparallel normal direction, J. Differential Geometry 8 (1973) 589-597.

[3] __, Pseudo-umbilical submanifolds of codimension 3 with constant mean curvature, Kodai Math. Sem. Rep. 25 (1973) 490-501.

[4] __, Submanifolds umbilical with respect to a quasi-parallel normal direction, Tensor 27 (1973) 41-44.

[5] M. Dajczer, Rigidity of isometric immersions of higher codimension, preprint. 
[6] M. Dajczer \& R. Tojeiro, Isometric immersions and the generalized Laplace and SinhGordon equations, preprint.

[7] M. do Carmo \& M. Dajczer, Riemannian metrics induced by two immersions, Proc. Amer. Math. Soc. 86 (1982) 115-120.

[8] _ Rotation hypersurfaces in spaces of constant curvature, Trans. Amer. Math. Soc. 227 (1983) 685-709.

[9] __, Conformal rigidity, Amer. J. Math. 109 (1987) 963-985.

[10] M. do Carmo, M. Dajczer \& F. Mercuri, Compact conformally flat hypersurfaces, Trans. Amer. Math. Soc. 288 (1985) 189-203.

[11] $\mathrm{J}$. Erbacher, Isometric immersions of constant mean curvature and triviality of the normal connection, Nagoya Math. J. 45 (1972) 139-165.

[12] W. Henke, Über die isometrischer Fortsetzbarkeit isometrischer Immersionen der Standard-m-Sphäre $S^{m}\left(\subset \mathbf{R}^{m+1}\right)$ in $\mathbf{R}^{m+2}$, Math. Ann. 219 (1976) 261-276.

[13] J. Lafontaine, Conformal geometry from the Riemannian viewpoint, Aspects of Mathematics, E 12, Vieweg, Braunschweig, 1988.

[14] J. D. Moore, Submanifolds of constant positive curvature. I, Duke Math. J. 44 (1977) 449-484.

[15] _ _ Isometric homotopy in codimension two, Trans. Amer. Math. Soc. 292 (1985) 653-663.

[16] B. O'Neill, Umbilics of constant curvature immersions, Duke Math. J. 32 (1965) 149160.

[17] T. Otsuki, Pseudo-umbilical submanifolds with $M$-index $\leq 1$ in Euclidean space, Kodai Math. Sem. Rep. 20 (1968) 296-304.

[18] L. Reckziegel, On the eigenvalues of the shape operator of an isometric immersion into a space of constant curvature, Math. Ann. 243 (1979) 71-82.

[19] L. Whitt, Isometric homotopy and codimension two isometric immersions of the n-sphere into Euclidean space, J. Differential Geometry 14 (1979) 295-302.

[20] K. Yano \& S. Ishihara, Pseudo-umbilical submanifolds of codimension 2, Kodai Math. Sem. Rep. 21 (1969) 365-382.

\section{IMPA, BRAZIL}

UNIVERSIDADE FEdERAL DE UBERLÂNDIA, BrazIL 